\title{
七甲川菁染料 $\mathbf{H}$-聚集体薄膜的线性及三阶非线性 光学性能研究
}

\author{
元以中 ${ }^{(2)}$ 康海峰 $^{(1} \quad$ 孙真荣 $^{(1 *}$ 王祖费 $^{(1}$ \\ (1) 光谱学与波谱学教育部重点实验室, 华东师范大学物理系, 上海 200062; (2) 华东理工大学材料科学与工程学院, 上海 200237. \\ *联系人, E-mail: zrsun@phy.ecnu.edu.cn)
}

摘要 采用旋涂法制备了一种七甲川菁染料的超薄膜, 利用原子力显微和紫外-可见吸收光谱等技术对 膜的形貌和线性光谱性质进行了研究表征, 结果表明染料在薄膜中形成了有序而稳定的 $\mathrm{H}$-聚集体. 前 向简并四波混频技术研究表明由于分子的聚集作用引起三阶非线性极化率的增大，利用集体谐振子模 型初步分析和讨论了产生非线性效应增强的内在机制.

关键词 旋涂膜 $\mathbf{H}$-聚集体 三阶非线性光学

有序分子聚集体的构筑和它们的非线性光学特 性是当今分子光电子学领域的一个非常活跃的研究 热点 ${ }^{[1,2]}$. 菁染料作为一类高性能的多功能生色团, 近年来, 人们对这类分子和它们的聚集体的光电和 非线性光学性能的研究已显示出极大的兴趣 ${ }^{[3,4]}$, 大 量理论和实验的研究结果表明, 与其单体相比, 菁染 料的 J-聚集体表现出更高的稳定性和更加优异的非 线性光学特性 ${ }^{[5 \sim 7]}$, 因而有望在未来的全光开关、全 光计算和光学逻辑门等光子型器件的研究和开发中 得到广泛应用. 与此形成鲜明对比的是, 对于菁染料 的另一类聚集体一 $\mathrm{H}$ - 聚集体的非线性光学的研究 报道目前国内外却相当有限, 这可能主要归因于它 们结构的不稳定性以及制备上的困难, 而这方面的 系统研究将有助于我们深入揭示不同聚集体的结构、 排列方式和有序程度与其宏观非线性光学特性间的 关系，从而为实现它们的材料化和制备高性能的分 子光子器件奠定基础. 基于此, 本文首先通过简单的 旋涂技术成功地制备了一种性能相当稳定的七甲川 菁染料 $\mathrm{HC}$ 的 $\mathrm{H}$-聚集体的超薄有序膜, 在对它的形貌 与结构进行充分表征的基础上，采用时间分辨的前 向简并四波混频技术测量了它的三阶非线性光学极 化率, 并初步分析了聚集体结构对三阶非线性光学 性能的影响.

\section{1 实验}

本实验所用的七甲川菁染料 HC 参照文献[3]方法
合成, 其结构经红外光谱、核磁共振氢谱、质谱和元 素分析确证, 如图 1.2,2,3,3-四氟-1-丙醇(tetra-fluoropropanol, TFP, $>99.9 \%$ )购于Acros Organics公司，使 用前末经纯化. 采用直径为 $30 \mathrm{~mm}$ 的双面抛光 $\mathrm{BK}-7$ 玻璃为基底, 基片在使用前按如下方法处理: 丙酮中 超声处理 $30 \mathrm{~min}$ 后, 依次用铬酸洗液和无水乙醇洗 涤, 最后用大量去离子水冲洗并用干燥的 $\mathrm{N}_{2}$ 流吹干. 为制备HC的旋涂膜, 将HC配成浓度为 $30 \mathrm{~g} / \mathrm{L}$ 的TFP 溶液, 超声振荡使其充分溶解, 经 $0.22 \mu \mathrm{m}$ 过滤头过 滤, 用 CHEMAT KW24A型旋转涂膜机甩涂在 BK-7 玻璃基片上, 控制合适的转速和时间即可获得高质 量的 HC的均匀固态薄膜.

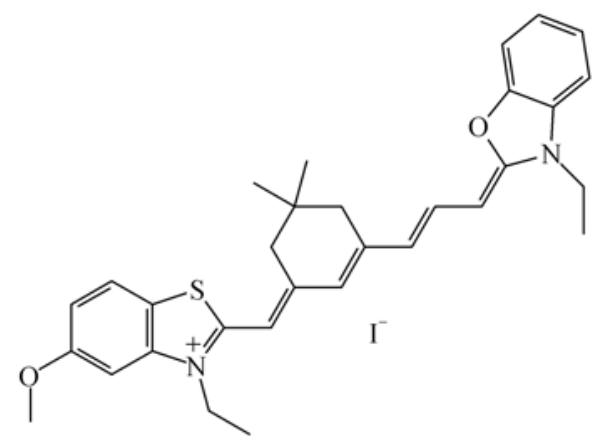

图 1 七甲川菁染料 $\mathrm{HC}$ 的分子结构

利用 SPA300HV 型原子力显微镜(AFM)进行薄 膜形貌表征. 采用 Hitachi U-3000型紫外-可见光谱仪 测定 HC 的溶液和薄膜的 UV-Vis 吸收光谱. 膜厚用 
ZD-003 型椭圆偏振仪测定.

采用前向简并四波混频(forward-DFWM)技术研 究待测样品的三阶非线性光学性能, 实验装置如图 2. 光源为 $\mathrm{Nd}$ : $Y A G$ 激光器的倍频光(输出波长 $532 \mathrm{~nm}$ ), 脉宽 $35 \mathrm{ps}$, 重复频率 $10 \mathrm{~Hz}$, 单脉冲能量 $3.0 \mathrm{~mJ}$. 激 光经 2 个小孔准直后, 被两块半透半反镜 $\mathrm{BS}_{2}$ 和 $\mathrm{BS}_{3}$ 分 成光强近似相等的 3 束光 $k_{1}, k_{2}$ 和 $k_{3}$. 经由延迟线 $\mathrm{G}_{1}$, $\mathrm{G}_{2}$ 和透镜 $\mathrm{L}$ 聚焦后入射到样品上. 3 束光相对于透镜 光轴的夹角约为 $2^{\circ}$. 在 $k_{4}$ 方向接收到共轭信号, 该信 号经光阑消除杂散光后, 由光电二极管 $\mathrm{PD}_{2}$ 接收, 并送 入取样积分器Boxcar和计算机进行处理.

实验中, 调节光路使 $k_{1}, k_{2}$ 和 $k_{3}$ 同时到达样品, 记 录产生的共轭信号 $I_{4}$. 在同样的实验条件下, 测量参 考样品 $\left(\mathrm{CS}_{2}\right)$ 所产生的共轭信号强度 $I_{4 r}$, 由下式求得 待测样品的三阶非线性极化率 $\chi^{(3)}$ 和非线性折射率 $n_{2}$ :

$$
\begin{gathered}
\chi^{(3)=\sqrt{\frac{I_{4}}{I_{4 r}}}} \frac{l_{r}}{l}\left(\frac{n}{n_{r}}\right)^{2}\left\{\frac{a \mathrm{e}^{\frac{1}{2} a l}}{1-\mathrm{e}^{-a l}}\right\} \chi_{r}^{(3)}, \\
n_{2}=\frac{12 \pi}{n} \chi^{(3)},
\end{gathered}
$$

式中 $\alpha$ 是样品的线性吸收系数, $n$ 是线性折射率, $l$ 为相 互作用长度, 下标 $r$ 代表参考样品 $\mathrm{CS}_{2}$, 在 $532 \mathrm{~nm}$
处样品的 $n_{r}$ 和 $\chi_{r}^{(3)}$ 分别为 1.61 和 $6.8 \times 10^{-13} \mathrm{esu}^{[8]}$.

\section{2 结果与讨论}

\section{1 菁染料薄膜的形貌表征}

原子力显微技术是表征薄膜表面微结构与形貌 的一种非常有效的手段. 图 3 为本实验制备的厚度为 $346 \mathrm{~nm}$ 的菁染料 $\mathrm{HC}$ 的旋涂膜的平面及三维 AFM 图 像, 由图 3 可以看出薄膜表面只有非常细微的起伏, 仅为 $1 \mathrm{~nm}$ 左右, 说明该薄膜的表面平整程度已基本 达到分子水平, 在已报道的有机旋涂膜中这样平整 的结构是非常少见的. 此外, 我们还发现染料 HC 在 薄膜中以比较规整的颗粒形态存在, 且颗粒尺寸分 布较为均一, 约为 $50 \mathrm{~nm}$, 颗粒排列紧密, 边界清晰 可见. 根据以上信息, 可初步推断染料在旋涂膜中形 成了有序的聚集体结构.

\section{2 菁染料薄膜的线性吸收特性}

为了进一步表征染料 $\mathrm{HC}$ 在旋涂膜中的聚集形态, 我们对它在稀溶液和薄膜状态下的线性吸收特性进 行了比较, 结果如图 4, 其中虚线为 $\mathrm{HC}$ 在 $1 \times 10^{-5}$ $\mathrm{mol} / \mathrm{L}$ 无水乙醇溶液(通光厚度为 $1 \mathrm{~cm}$ )中的吸收曲线, 可近似认为是染料单体的光谱行为; 而实线是相应 的旋涂膜的吸收光谱. 由图 4 可知, 染料单体的峰值 吸收波长位于 $738 \mathrm{~nm}$ 处, 对应于分子由基态 $S_{0}$ 到第一

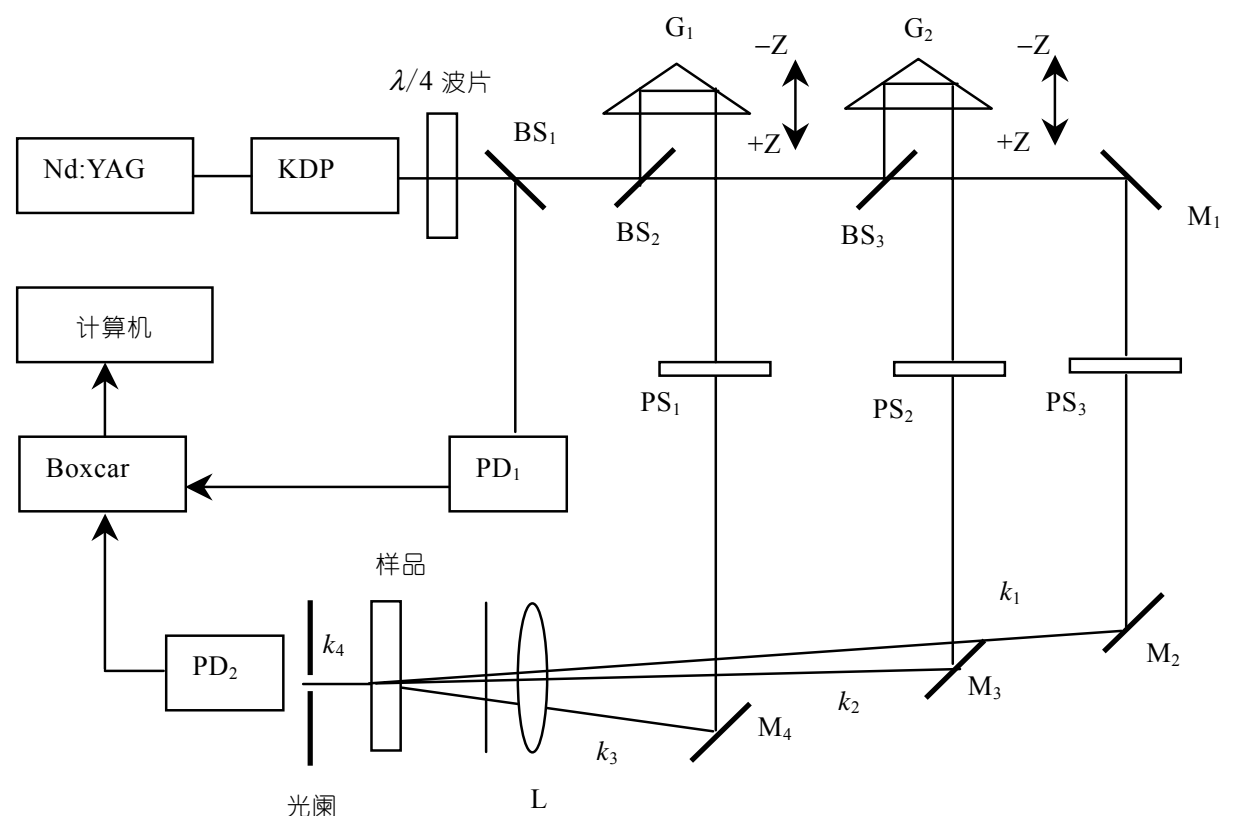

图 2 前向简并四波混频实验装置示意图 


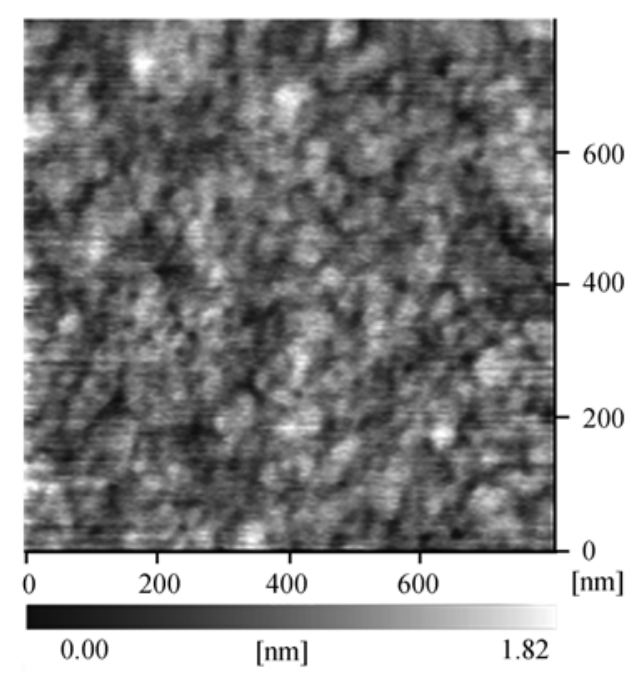

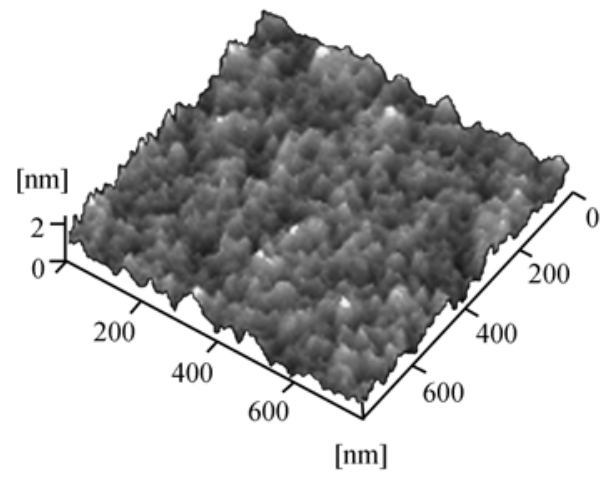

图 3 染料 $\mathrm{HC}$ 旋涂膜的平面及三维 $\mathrm{AFM}$ 照片

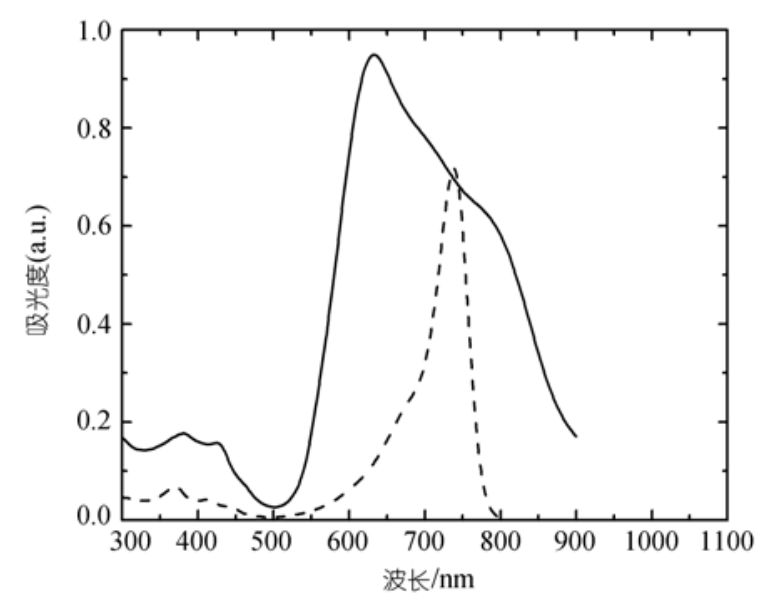

图 4 染料 $\mathrm{HC}$ 在无水乙醇稀溶液(虚线)和旋涂膜(实线)中 的紫外-可见吸收光谱

激发单重态 $S_{1}$ 的 $\pi-\pi{ }^{*}$ 轨道跃迁, 与之相比, 染料旋涂 膜的吸收波长发生了显著的蓝移, 其 $\lambda_{\text {max }}$ 为 $633 \mathrm{~nm}$, 且谱线出现了一定程度的展宽, 这说明染料在旋涂 膜中是以 $\mathrm{H}-$ 聚集体的形式存在的.

上述现象可从Kasha等人 ${ }^{[9]}$ 提出的一维Frenkel激 子耦合模型得到很好的解释, 当 $N$ 个分子因电偶极矩 的相互作用而彼此靠近时, 分子的 $N$ 重简并的第一激 发单重态 $S_{1}$ 将分裂为 $N$ 个非简并的单激子能级. 理论 计算表明，当分子中心的联线与分子跃迁偶极矩矢 量之间的夹角 $\theta>54.7^{\circ}$, 即分子间彼此平行且发生面 对面(face-to-face)的紧密堆砌( $\mathrm{H}$-聚集体)时, 由基态 向高能级的单激子态的跃迁几率将远大于向低能级 单激子态的跃迁几率, 同时由于激子能级的窄带隙
特性, 由后者产生的弱吸收带将被前者对应 的主吸收带湮没, 因此, 与单体相比, $\mathrm{H}$-聚集体在吸 收光谱上即表现为峰值吸收波长的蓝移和谱线的展 宽; 反之, 当 $\theta<54.7^{\circ}$, 即分子间以头-尾相接的方式 ( $\mathrm{J}$-聚集体)堆积时, 则只可能发生由基态向最低能级 的单激子态的跃迁, 因而在吸收光谱上应表现为一 个强而尖锐的、红移的峰值吸收. 根据上述模型, 同 时结合图 4 的实验结果和前面的AFM数据, 可以充 分肯定, 染料 $\mathrm{HC}$ 在旋涂的过程中自发形成了有序的 $\mathrm{H}$-聚集体.

\section{3 菁染料薄膜的三阶非线性光学特性}

我们分别测定了染料 $\mathrm{HC}$ 的旋涂膜及其无水乙醇 稀溶液 $\left(1 \times 10^{-5} \mathrm{~mol} / \mathrm{L}\right)$ 的三阶非线性光学参数, 测量 结果见表 1 . 由表 1 可见, 染料在形成 $\mathrm{H}$-聚集体后, 它的非线性光学响应大大增强, 在相同的测试条件 下, 其旋涂膜的 $\chi^{(3)}$ 和 $n_{2}$ 值比在溶液中提高了一个数 量级.

表 1 染料 $\mathrm{HC}$ 在无水乙醇稀溶液和旋涂膜中的 线性及三阶非线性光学参数

\begin{tabular}{rccc}
\hline & $\lambda_{\max } / \mathrm{nm}$ & $\chi^{(3)} / \mathrm{esu}$ & $n_{2} / \mathrm{esu}$ \\
\hline $\mathrm{HC}$ 溶液 & 738 & $3.31 \times 10^{-13}$ & $9.15 \times 10^{-12}$ \\
$\mathrm{HC}$ 薄膜 & 633 & $2.21 \times 10^{-12}$ & $3.2 \times 10^{-11}$ \\
\hline
\end{tabular}

$\mathrm{HC}$ 分子可看作由电子给体(electron-donor，D)和 电子受体 (electron-acceptor, A) 经共轭七甲川链共价 连接的D- $\pi$-A体系, 它们与激光场的超快相互作用会 导致分子中不同位置的 $\pi$ 电子密度的瞬时变化, 由此 
产生一系列光诱导的电子-空穴对(光生激子) ${ }^{[8]}$. 按照 Schulz和Tretiak等人 ${ }^{[10,11]}$ 建立的电子体系的集体谐振 子(CEO)模型, 我们认为分子聚集体的三阶非线性光 学响应来源于其内部产生的所有光生激子的非谐振 动的贡献之和, 它的三阶非线性极化率 $\chi^{(3)}$ 的数值则 在一定空间范围内与光生激子的特征相干尺寸 $L_{\rho}^{(3)}$ 有 关, $L_{\rho}^{(3)}$ 由下式给出 ${ }^{[10]}$ :

$$
L_{\rho}^{(3)} \equiv\left[N_{0} \sum_{m, n}\left|\rho_{m n}\right|^{2}\right]^{-1}\left[\left(\sum_{m, n}\left|\rho_{m n}\right|\right)^{2}\right],
$$

式中, $N_{0}$ 是构成聚集体的分子总数, $\rho_{m n}$ 为分子聚集 体在外光场激励下的含时单激子密度矩阵, 它由相 应的定态项 $\bar{\rho}_{m n}$ 和光诱导的多阶含时项 $\delta \rho_{m n}^{(k)}(t)$ 共同 组成, 可定量表达如下 ${ }^{[10]}$ :

$$
\rho_{m n}(t)=\bar{\rho}_{m n}+\delta \rho_{m n}^{(1)}(t)+\delta \rho_{m n}^{(2)}(t)+\delta \rho_{m n}^{(3)}(t)+\ldots,
$$

其中 $\delta \rho_{m n}^{(k)}(t)$ 对应于 $\rho_{m n}$ 在外场作用下的 $k$ 阶贡献, 本 研究中 $k=3$. 由(3)和(4)式可知 $\chi^{(3)}$ 随 $L_{\rho}^{(3)}$ 的增大而增 大, 且 $L_{\rho}^{(3)}$ 在 1 到 $N_{0}$ 的范围内变化, 其具体取值主要 依赖于聚集体中分子排列的有序程度和聚集体的分 子密度 $[12,13]$. 本实验中, 由于 $\mathrm{HC}$ 分子在稀溶液中的 分布是无序的, 而且单位体积的 $\mathrm{HC}$ 分子数目有限, 于是光生激子仅能在单个分子空间离域, 即 $L_{\rho}^{(3)}$ 较小, 因此难以形成分子间的非线性光学响应. 而当 $\mathrm{HC}$ 的 聚集状态由稀溶液转变为有序的 $\mathrm{H}$-聚集体薄膜后, 它的分子密度和分子排列的规整性都将大大增加, 这样体系在外光场激励下产生的光生激子不仅存在 于分子内部, 还将在组成 $\mathrm{H}$-聚集体的部分或全部分 子间离域, 引起 $L_{\rho}^{(3)}$ 的显著增加, 使体系产生相干的 非线性光学响应, 进而导致 $\chi^{(3)}$ 的大幅度提高. 此外, 由于HC分子两端电子给体和受体取代基的静电相互 作用在 $\mathrm{H}$-聚集体内部形成了一定强度的库仑势场, 这也能在一定程度上提高光生激子的分离效率并加 快它们在聚集体中的传输速度, 从而对 $\chi^{(3)}$ 的增加起 到积极的作用.

\section{3 结论}

通过控制合适的成膜条件，制备了七甲川菁染
收光谱对膜的形貌和结构进行了详细的表征, 皮秒 forward-DFWM技术测量了三阶非线性极化率 $\chi^{(3)}$ 和 非线性折射率 $n_{2}$ 等重要参数, 研究表明微结构的有序 性和分子密度的显著增加引起 $\mathrm{H}-$ 聚集体非线性响应 的大大增强, 且该增强效应主要来源于光生激子在 分子聚集体空间内的强烈离域作用.

\section{参考文献}

1 Stupp S I, LeBonheur V, Walker K, et al. Supramolecular materials: Self-organized nanostructures. Science, 1997, 276: 384-389[DOI]

2 Li H M, Zhou Y S, Li Y L, et al. Fabrication and nonlinear optical properties of ultrathin films containing sulfonate functionalized fullerenes. Chem Phys Lett, 2004, 383: 230-234[DOI]

3 Mishra A, Behra R K, Behra P K, et al. Cyanines during the 1990s: A review. Chem Rev, 2000, 100: 1973-2011[DOI]

4 Ushiroda S, Ruzycki N, Lu Y, et al. Dye sensitization of the anatase (101) crystal surface by a series of dicarboxylated thiacyanine dyes. J Am Chem Soc, 2005, 127: 5158-5168[DOI]

5 Ohta K, Yang M, Fleming G R. Ultrafast exciton dynamics of $\mathrm{J}$-aggregates in room temperature solution studied by third-order nonlinear optical spectroscopy and numerical simulation based on exciton theory. J Chem Phys, 2001, 115: 7609-7621[DOI]

6 Markov R V, Plekhanov A I, Shelkovnikov V V, et al. Nonlinear optical properties of one-dimensional organic molecular aggregates in nanometer films. Microelectron Eng, 2003, 69: 528-531[DOI]

7 Vannikov A V, Grishina A D, Shapiro B I, et al. Photoelectric, nonlinear optical and photorefractive properties of polyimide doped with J-aggregates of cyanine dye. Chem Phys, 2003, 287: 261271[DOI]

8 Brédas J L, Adant C, Tackx P, et al. Third-order nonlinear optical response in organic materials: Theoretical and experimental aspects. Chem Rev, 1994, 94: 243-278[DOI]

9 Kasha M, Rawls H R, El-Bayoumi M A. The exciton model in molecular spectroscopy. Pure Appl Chem, 1965, 11: 371-392

10 Schulz M, Tretiak S, Chernyak V, et al. Size scaling of third-order off-resonant polarizabilities. Electronic coherence in organic oligomers. J Am Chem Soc, 2000, 122: 452-459[DOI]

11 Tretiak S, Mukamel S. Density matrix analysis and simulation of electronic excitations in conjugated and aggregated molecules. Chem Rev, 2002, 102: 3171-3212[DOI]

12 Meier T, Zhao Y, Chernyak V, et al. Polarons, localization, and excitonic coherence in superradiance of biological antenna complexes. J Chem I Phys, 1997, 107: 3876-3893[DOI]

13 Subramanian V, Evans D G. Excitation energy transfer in model light-harvesting antennae. J Phys Chem B, 2004, 108: 10851095[DOI] 